\title{
BMJ Open Regional versus General Anesthesia for Promoting Independence after Hip Fracture (REGAIN): protocol for a pragmatic, international multicentre trial
}

Mark D Neuman, ${ }^{1,2,3,4}$ Susan S Ellenberg, ${ }^{5}$ Frederick E Sieber, ${ }^{6}$ Jay S Magaziner, ${ }^{7}$ Rui Feng, ${ }^{5}$ Jeffrey L Carson, ${ }^{8}$ and the REGAIN Investigators

To cite: Neuman MD, Ellenberg SS, Sieber FE, et al. Regional versus General Anesthesia for Promoting Independence after Hip Fracture (REGAIN): protocol for a pragmatic, international multicentre trial. BMJ Open 2016;6:e013473. doi:10.1136/bmjopen-2016013473

- Prepublication history and additional material is available. To view please visit the journal (http://dx.doi.org/ 10.1136/bmjopen-2016013473).

Received 13 July 2016 Revised 6 September 2016 Accepted 15 September 2016

CrossMark

For numbered affiliations see end of article.

Correspondence to Dr Mark D Neuman; neumanm@mail.med.upenn. edu

\section{ABSTRACT}

Introduction: Hip fractures occur 1.6 million times each year worldwide, with substantial associated mortality and losses of independence. At present, anaesthesia care for hip fracture surgery varies widely within and between countries, with general anaesthesia and spinal anaesthesia representing the 2 most common approaches. Limited randomised evidence exists regarding potential short-term or long-term differences in outcomes between patients receiving spinal or general anaesthesia for hip fracture surgery.

Methods: The REGAIN trial (Regional vs General Anesthesia for Promoting Independence after Hip Fracture) is an international, multicentre, pragmatic randomised controlled trial. 1600 previously ambulatory patients aged 50 and older will be randomly allocated to receive either general or spinal anaesthesia for hip fracture surgery. The primary outcome is a composite of death or new inability to walk 10 feet or across a room at 60 days after randomisation, which will be assessed via telephone interview by staff who are blinded to treatment assignment. Secondary outcomes will be assessed by in-person assessment and medical record review for in-hospital end points (delirium; major inpatient medical complications and mortality; acute postoperative pain; patient satisfaction; length of stay) and by telephone interview for 60-day, 180-day and 365-day end points (mortality; disability-free survival; chronic pain; return to the prefracture residence; need for new assistive devices for ambulation; cognitive impairment).

Ethics and dissemination: The REGAIN trial has been approved by the ethics boards of all participating sites. Recruitment began in February 2016 and will continue until the end of 2019. Dissemination plans include presentations at scientific conferences, scientific publications, stakeholder engagement efforts and presentation to the public via lay media outlets. Trial registration number: NCT02507505, Preresults.
Strengths and limitations of this study

- The REGAIN trial (Regional vs General Anesthesia for Promoting Independence after Hip Fracture) will evaluate outcomes of common anaesthesia techniques for hip fracture surgery, an event that occurs over 1.6 million times each year worldwide, through an international, multicentre, randomised trial.

- Pragmatic, 'real-world' treatment protocols are reflective of current practice and will allow the results to be generalised across a range of care settings.

- Collection of patient-centred outcomes data including measures of functional independence, at up to 1 year will provide insight into the relationship between the study intervention and meaningful patient end points.

- Input by patients and stakeholders at each stage will improve translation and dissemination of eventual results to affected communities.

- Data collection for certain in-hospital adverse events will rely on medical record review; as such, events that are not recorded in the medical record may not be captured.

\section{INTRODUCTION}

Over 1.6 million hip fractures occur each year worldwide, with major consequences for the individual and society. ${ }^{1}{ }^{2}$ Within 12 months of fracture, $25 \%$ of patients die, ${ }^{34}$ and half of previously community-dwelling patients either die or require new nursing home admission. ${ }^{5}$ Hip fractures create substantial needs for informal caregiving ${ }^{6} 7$ and postacute and long-term care involving major costs to society; ${ }^{8}$ the estimated costs attributable to hip fractures in the USA exceeded $\$ 12$ billion in 2005 and will exceed $\$ 18$ billion by $2025 .^{9}$ 
Nearly all patients with hip fractures undergo surgical treatment. Anaesthesia for hip fracture surgery varies widely in practice, ${ }^{10}{ }^{11}$ with general anaesthesia and spinal anaesthesia representing the two most common approaches. ${ }^{12}$ Available studies comparing outcomes with spinal versus general anaesthesia for hip fracture surgery have been reviewed elsewhere. ${ }^{13-17}$ While spinal anaesthesia has been theorised to improve outcomes by avoiding the need for tracheal intubation and exposure to general anaesthetics, available randomised studies have yielded equivocal findings regarding the relative superiority of one technique over the other with regard to either short-term morbidity and mortality or longer term functional recovery.

Existing randomised studies are characterised by major shortcomings. A 2016 Cochrane review of trials comparing spinal versus general anaesthesia for hip fracture surgery between 1977 and 2012 rated the quality of available evidence as 'very poor' for all outcomes studied. ${ }^{13}$ A 2011 systematic review by the UK Clinical Guideline Centre concluded that "no recent randomised trials were identified that fully address' the clinical effectiveness of regional versus general anaesthesia for hip fracture surgery, and that the available evidence "is old and does not reflect current practice'. ${ }^{18}$ In particular, few data are available to characterise the impact of anaesthesia technique on patient-centred outcomes, such as functional recovery or satisfaction.

\section{Study objectives}

The REGAIN trial (Regional vs General Anesthesia for Promoting Independence after Hip Fracture) will evaluate the effect of spinal versus general anaesthesia on recovery of ambulation at 60 days after randomisation (primary outcome) and other patient-centred outcomes measured at up to 1 year. Our primary hypothesis is that patients who receive spinal anaesthesia will demonstrate improved ambulation at 60 days after randomisation compared with patients who receive general anaesthesia. The membership of the REGAIN investigator group is described in the online supplementary appendix.

\section{METHODS AND ANALYSIS \\ Study design}

We will perform a randomised, multicentre, pragmatic active comparator study of two standard care approaches to anaesthesia for hip fracture surgery (ie, spinal and general anaesthesia). Study end points will be assessed via in-person interview (during hospitalisation), medical record review, telephone interview (after hospital discharge) and a vital records database search. The primary outcome will be assessed at 60 days after randomisation by a telephone interviewer blinded to treatment assignment. As noted below, all postdischarge outcomes (including the primary end point) will be assessed in a blinded fashion; however, to increase the feasibility of trial implementation across sites with varied staffing capabilities, we will encourage but not require those staff that will assess in-hospital end points to be blinded to treatment assignment.

Pragmatic design features of the REGAIN trial: The development process for the REGAIN trial protocol engaged patients, stakeholders, researchers and clinicians to develop a pragmatic study design that would yield findings with relevance to clinical practice across a range of settings. We used the PRECIS tool ${ }^{19}$ to formalise the implications of specific design choices for the nature of the REGAIN trial as a pragmatic (effectiveness) trial versus an explanatory (efficacy) trial (table 1) across a range of domains.

Eligibility criteria appear in box 1.

\section{Baseline assessment}

As shown in the study assessment schedule (table 2), enrolled patients will undergo a prerandomisation assessment that includes a medical history questionnaire, a brief medical record review, and selected assessments to assess: (1) prefracture disability, as measured by the 12-item WHO Disability Assessment Schedule, V.2.0 (WHODAS 2.0), a validated measure that assesses cognition, mobility, self-care, interpersonal relationships, work and household roles, and participation in society ${ }^{20} 21$ Melbourne, Australia: Alfred Hospital, 2014); notably, as we are unable to measure prefracture disability prospectively in this population, we will rely on patient recall of prefracture self-performance in WHODAS V.2.0 domains; (2) cognitive status at the time of interview, as measured by the Short Blessed Test, a well-validated brief cognitive screening tool ${ }^{22}{ }^{23}$ (3) delirium at the time of interview, as measured by the $3 \mathrm{~min}$ assessment for Confusion Assessment Method-defined delirium (3D-CAM), a well-validated brief assessment tool with high sensitivity and specificity for delirium; $;^{24}{ }^{25}(4)$ prefracture pain symptoms, as measured by items adapted from the Brief Pain Inventory; ${ }^{26}{ }^{27}$ and (5) resilience at the time of interview, as measured by the Brief Resilience Scale, a short, validated tool measuring an individual's ability to 'bounce back' from a stressful event. $^{28}$ We will collect contact information for the patient and for alternate contacts as required for telephone follow-up. In patients who agree to provide these data, social security numbers and Medicare beneficiary identifiers will be collected for relevant database linkages.

\section{Interventions}

We will randomly allocate patients to receive standard care spinal anaesthesia or standard care general anaesthesia. Apart from the decision regarding the primary anaesthetic technique (spinal vs general anaesthesia), all decisions about preoperative, intraoperative and postoperative care will be made by the clinical care team.

The intervention will occur by providing the treating clinical anaesthesia staff written instructions (box 2) directing them to perform a standard care spinal 
Table 1 Pragmatic design features of the REGAIN trial

\section{PRECIS domain(s)}

1. Participant eligibility criteria

2 and 3. Experimental and comparison interventions-flexibility

4 and 5. Experimental and comparison interventions-practitioner expertise

\section{Follow-up intensity}

\section{Primary trial outcome}

8. Participant compliance with prescribed intervention

\section{Practitioner adherence to study protocol}

10. Analysis of primary outcome

\section{Assessment}

Ghis study will enrol a broad group of hip fracture patients without contraindications to regional or general anaesthesia who were ambulatory prior to fracture. Patients will be enrolled from a group of diverse academic and community sites. While the results may not be generalisable to some groups of patients, such as those who were not ambulatory before fracture, the broad eligibility criteria make the study highly pragmatic in this domain. Treating physicians will receive brief, simple and highly flexible care protocols for patients randomised to receive spinal anaesthesia; these protocols will state explicitly that co-interventions will are permitted based on clinical judgement. The study is maximally pragmatic in this domain. Study protocols will be administered by clinical anaesthesia staff without requirements for additional training in specific anaesthesia techniques or advanced expertise. The study is maximally pragmatic in this domain. In-hospital outcomes will be assessed by 3 brief assessments over the first 3 postoperative days and by chart review at discharge. Blinding will not be required for in-hospital assessments to maximise study feasibility across a range of hospital settings. Postdischarge follow-up will occur via brief minute phone interviews at 60,180 and 365 days by assessors who are blinded to treatment assignment. Survival will be assessed by searches of vital records files. The study is highly pragmatic in this domain.

The primary outcome (death or inability to walk across a room at 2 months) is simple and pragmatic; secondary outcomes are also pragmatic end points, including overall disability, return to prefracture residence and all-cause mortality.

Randomisation to regional vs general anaesthesia will be clearly stated in the study consent form. Since patients who do not want either regional or general anaesthesia will be unlikely to enrol in the trial, the study is more explanatory than pragmatic in this domain.

Practitioner adherence to treatment assignment will be monitored and efforts will be made to limit deviations from assigned treatments; the study is more explanatory than pragmatic in this regard.

All randomised patients will be included in the primary analysis; additional analyses will be adjusted for compliance with the study protocol. A priori subgroups will be examined; the proposal is moderately pragmatic in this regard.

Domains are adapted from the Pragmatic-Explanatory Continuum Indicator Summary (PRECIS) framework of Thorpe et al..$^{19}$ The 10 listed domains for the REGAIN trial are described and characterised in relation to design aspects common to pragmatic (effectiveness) versus explanatory (efficacy) trials.

anaesthetic or a standard care general anaesthetic. For patients who are randomised to receive spinal anaesthesia, instructions will be provided to titrate any sedation to maintain arousability to tactile stimulus or voice, and to rate the level of sedation in the anaesthetic record at least once between induction and emergence on a scale of 1 (deep sedation) to 5 (alert) based on the arousability subscale of the Observer's Assessment of Alertness/ Sedation scale. ${ }^{29}$

\section{Outcomes}

Primary outcome: independence in walking at 60 days after randomisation: The primary outcome will be assessed by telephone interview at 60 days after randomisation. This assessment will be conducted centrally by the study Clinical Coordinating Center at the University of Pennsylvania. Assessments will be conducted by the staff who will be blinded to treatment assignment. Patients who report being unable to walk 10 feet or across a room without human assistance, or who die within 60 days of fracture will be classified as treatment failures. For patients who are unable to provide their own responses, available secondary informants will be interviewed regarding the participant's ability to walk independently at 60 days.

The primary outcome for REGAIN was selected based on consultation with patient and stakeholder partners as a clinically meaningful measure that also predicts key long-term outcomes. Data from the Baltimore Hip Studies indicate that patients who were unable to walk at 60 days demonstrated high rates of persistent inability to walk at 1 year (OR 11.1, 95\% CI 6.6 to 18.7), 1 year mortality (OR 3.6, 95\% CI 1.9 to 6.5 ) and new nursing home placement at 1 year (OR 6.2, 95\% CI 3.9 to 9.7) compared with those who could walk independently at 60 days. ${ }^{30}$ 
Box 1 Inclusion and exclusion criteria for the REGAIN (Regional versus General Anesthesia for Promoting Independence after Hip Fracture) trial

\section{Inclusion criteria}

- Clinically or radiographically diagnosed intracapsular or extracapsular hip fracture;

- Planned surgical treatment via hemiarthroplasty, total hip arthroplasty or appropriate fixation procedure;

- Age $\geq 50$ years;

- Ability to walk 10 feet or across a room without human assistance before fracture.

Exclusion criteria

- Planned concurrent surgery not amenable to spinal anaesthesia;

- Absolute contraindications to spinal anaesthesia, including: (1) known or suspected congenital or acquired coagulopathy; (2) active use of pharmacological anticoagulants within a timeframe defined to contraindicate neuraxial block placement by available American Society of Regional Anesthesia guidelines (2) known or suspected unrepaired critical or severe aortic stenosis; (3) known or suspected active skin infection at the planned needle insertion site; (4) known or suspected elevated intracranial pressure contraindicating dural puncture;

- Patient is known or suspected to be at elevated risk for malignant hyperthermia;

- Periprosthetic fracture;

- Prior participation in the REGAIN trial;

Prisoner status;

- Determination by the attending surgeon, the attending anaesthesiologist, or the site clinical director or their designate, that the patient would not be suitable for randomisation.

Our selection of the primary outcome for REGAIN was also informed by the successful use of the same end point in the FOCUS trial, a 2100-patient randomised trial compared two different transfusion strategies after hip fracture surgery. ${ }^{30}$ The use of telephone follow-up was chosen based on the past successful use of this approach in prior studies, ${ }^{31-33}$ as well as to allow for a high degree of standardisation and quality assurance for outcome data collection across a diverse group of institutions, potentially including those with limited access to research staff.

Secondary outcomes (in-hospital): (1) Postoperative delirium will be assessed by study staff prior to randomisation and daily from postoperative day 1 through postoperative day 3 or the day of discharge (whichever occurs first) via the 3D-CAM; ${ }^{24}{ }^{25}$ (2) acute postoperative pain will be assessed by study staff via in-person interview daily from postoperative day 1 through postoperative day 3 or the day of discharge (whichever occurs first) via items adapted from the Brief Pain Inventory; ${ }^{26} 27$ (3) satisfaction with anaesthesia care will be assessed on postoperative day 3 or the day of discharge (whichever occurs first) via the Bauer Patient Satisfaction Questionnaire; ${ }^{34}$ (4) inpatient mortality and major inpatient morbidity will be assessed via chart review by site staff using standardised outcome definitions following hospital discharge, death or at 30 days after surgery, whichever occurs first. To increase the feasibility of trial implementation across diverse sites, we will encourage but not require those staff that will assess in-hospital end points to be blinded to treatment assignment.

Secondary outcomes (postdischarge): Secondary outcomes will be collected via telephone interview by blinded study staff at 60,180 and 365 days after randomisation. Secondary outcomes will include: (1) overall health and disability, as assessed via telephone interview with patients or proxies via the WHODAS V.2.0 ${ }^{20}$ (2) chronic pain, as assessed via two adapted Brief Pain Inventory items to assess the extent of pain at worst and on average over the past 7 days; (3) cognitive function, as assessed by the Short Blessed Test; ${ }^{22} 23$ (4) independence in locomotion and need for assistive devices for walking (ie, cane, walker); and (5) location of residence (ie, home vs nursing facility). Finally, vital status will be assessed via patient and/or proxy telephone interview at $\sim 60,180$ and 365 days after randomisation and via a National Death Index (NDI) search for US patients in the final year of the study.

\section{Sample size planning}

The REGAIN trial will randomise 1600 patients to spinal versus general anaesthesia for hip fracture surgery. Assuming a $34 \%$ rate of the primary outcome (death or new inability to walk at 60 days) in the general anaesthesia arm (the rate observed in the 2000-patient FOCUS trial), ${ }^{33}$ this sample will provide $80 \%$ power to detect a relative risk of 0.78 for the primary outcome among patients receiving spinal versus general anaesthesia and $90 \%$ power to detect a relative risk of 0.76 at an $\alpha$ value of 0.05 . Sample size calculations allow for $5 \%$ loss to follow-up for the primary outcome and a $5 \%$ cross-over rate from spinal to general anaesthesia based on available published data on rates of spinal anaesthetic failures in clinical practice. ${ }^{35-37}$

The planned sample will also provide sufficient power for testing of hypotheses related to secondary outcomes. In terms of overall health and disability, a change of eight points or greater represents a clinically important difference for the WHODAS V.2.0 $;^{20}$ the WHODAS V.2.0 SD among adults aged 75-85 with more than one chronic physical condition is $15.8 \%{ }^{38}$ Given these assumptions, our sample will provide over $99 \%$ power to detect a clinically significant difference in disability at 180 days between groups.

\section{Recruitment}

All participants will be recruited in hospital settings between the time of presentation and the time of surgery. Orthopaedic surgeons performing hip fracture surgery at each recruiting site will be contacted in advance of the initiation of study accrual to assess willingness for their patients to be enrolled. For potentially eligible patients, a member of the REGAIN research 
Table 2 Visit schedule for the REGAIN trial

\begin{tabular}{|c|c|c|c|c|c|c|c|c|c|c|c|}
\hline \multirow[b]{3}{*}{ Time point $^{*}$} & \multicolumn{11}{|l|}{ Study period } \\
\hline & \multirow{2}{*}{$\begin{array}{l}\text { Preallocation } \\
\text { Preoperative } \\
\left(-t_{1}\right) \\
\end{array}$} & \multirow{2}{*}{$\begin{array}{l}\text { Allocation } \\
\text { POD } 0\left(t_{0}\right)\end{array}$} & \multicolumn{8}{|c|}{ Postallocation } & \multirow{2}{*}{$\begin{array}{l}\text { Closeout } \\
\text { POD } 365 \\
\pm 60\left(t_{9}\right)\end{array}$} \\
\hline & & & $\begin{array}{l}\text { POD } 0 \\
\left(t_{1}\right)\end{array}$ & $\begin{array}{l}\text { POD } 1 \\
\left(t_{2}\right)\end{array}$ & $\begin{array}{l}\text { POD } 2 \\
\left(t_{3}\right)\end{array}$ & $\begin{array}{l}\text { POD 3 } \\
\left(\mathrm{t}_{4}\right)\end{array}$ & $\begin{array}{l}\text { POD } 30 \\
\left(t_{5}\right)\end{array}$ & $\begin{array}{l}\text { POD 60 } \\
\pm 30\left(t_{6}\right) \\
\end{array}$ & $\begin{array}{l}\text { POD } 180 \\
\pm 45\left(\mathrm{t}_{7}\right)\end{array}$ & $\begin{array}{l}\text { POD } 365 \\
\pm 60\left(t_{8}\right) \\
\end{array}$ & \\
\hline \multicolumn{12}{|l|}{ Enrolment } \\
\hline Eligibility & $\mathrm{x}$ & & & & & & & & & & \\
\hline Informed consent & $\mathrm{x}$ & & & & & & & & & & \\
\hline Allocation & & $\mathrm{x}$ & & & & & & & & & \\
\hline Intervention & & & $\mathrm{x}$ & & & & & & & & \\
\hline \multicolumn{12}{|l|}{ Assessments } \\
\hline Medical history & $\mathrm{x}$ & & & & & & & & & & \\
\hline Locomotion ability & $\mathrm{x}$ & & & & & & & $\mathrm{x}$ & $\mathrm{x}$ & $\mathrm{x}$ & \\
\hline Pain scales & $\mathrm{x}$ & & & $\mathrm{x}$ & $x$ & $\mathrm{x}$ & & $\mathrm{x}$ & $\mathrm{x}$ & $\mathrm{x}$ & \\
\hline Short Blessed Test (cognition) & $\mathrm{x}$ & & & & & $\mathrm{x}$ & & $\mathrm{x}$ & $x$ & $\mathrm{x}$ & \\
\hline WHODAS V.2.0 (disability) & $\mathrm{x}$ & & & & & & & $\mathrm{x}$ & $\mathrm{x}$ & $\mathrm{x}$ & \\
\hline \multicolumn{12}{|l|}{ Assessments } \\
\hline 3D-CAM (delirium) & $\mathrm{x}$ & & & $x$ & $\mathrm{x}$ & $\mathrm{x}$ & & & & & \\
\hline Bauer Scale (satisfaction)† & & & & & & $\mathrm{x}$ & & & & & \\
\hline $\begin{array}{l}\text { Medical record review: } \\
\text { intraoperative and postoperative } \\
\text { eventsł }\end{array}$ & & & & & & & $\mathrm{x}$ & & & & \\
\hline $\begin{array}{l}\text { Mortality (medical record review/ } \\
\text { telephone follow-up) }\end{array}$ & & & & & & & $x$ & $x$ & $x$ & & \\
\hline Mortality (National Death Index) & & & & & & & & & & $\mathrm{x}$ & \\
\hline Study closeout & & & & & & & & & & & $\mathrm{x}$ \\
\hline
\end{tabular}


Box 2 Treatment regimens for the REGAIN (Regional versus General Anesthesia for Promoting Independence after Hip Fracture) trial

Instructions for patients randomised to receive spinal anaesthesia: please perform a single-shot spinal anaesthetic, with sedation as needed for block placement and intraoperative comfort. Please titrate any intraoperative sedation to maintain arousability to tactile stimulus or voice. Conversion to general anaesthesia is permitted if required by clinical circumstances. Please conduct all other aspects of anaesthesia care, including monitoring, medication selection and dosing, supplemental nerve blocks, and management of intraoperative events as per your usual routine.

Instructions for patients randomised to receive general anaesthesia: please perform a general anaesthetic. Please use an inhaled anaesthetic agent for maintenance and use intravenous opiates as needed for analgesia. Airway management may be via endotracheal tube, laryngeal mask airway or other device as dictated by clinical circumstances. Please conduct all other aspects of anaesthesia care, including monitoring, medication selection and dosing, supplemental nerve blocks and management of intraoperative events as per your usual routine.

team will approach the patient and/or their legally authorised representative (based on local Institutional Review Board (IRB) guidance) between the time of diagnosis and the time of surgery to explain the study, complete a brief screening evaluation, and obtain written informed consent. For patients who are too sick or who are not competent to give their own permission to enter the study, consent will be obtained from the patient's legally authorised representative if permitted by the local IRB. ${ }^{39}$

REGAIN recruiting sites have been selected to represent a broad range of geographic locations and practice settings in the USA and Canada, including large teaching and non-teaching hospitals and smaller community facilities. The site selection process for REGAIN included consideration of annual hip fracture volume, presence of buy-in from clinical leaders, research infrastructure and past experience with randomised trials.

\section{Allocation}

Randomisation will be carried out on the day of surgery immediately prior to start of anaesthesia care and will be performed centrally through an online electronic data management system after confirmation with the assigned anaesthesia and orthopaedic surgery providers that the patient is suitable for randomisation. Site research staff will obtain the randomisation assignment from the data management system web portal immediately prior to surgery and will communicate the treatment assignment to the anaesthesia team. Participants will be randomly assigned to one of the two treatment regimens in a 1:1 ratio. For each arm, balanced randomisation of participants, stratified by site, sex and fracture type (intracapsular vs extracapsular), will be achieved by permuted block randomisation with variable block sizes. ${ }^{40}{ }^{41}$ Participants will not be blinded to treatment assignment.

\section{Data analysis and management}

Both primary and secondary outcomes will be evaluated under the intention-to-treat principle. All hypothesis tests will be performed using a two-sided significance level (type I error) of $\alpha=0.05$. Sensitivity analyses using the actual treatment received (rather than assigned) will be performed and compared with the intention-to-treat analysis results; additional sensitivity analyses will assess the potential impact of missing data due to losses to follow-up.

The primary analysis will compare the proportions of patients who can walk independently at 60 days between groups randomised to spinal versus general anaesthesia using the Mantel-Haenszel tests, stratified by site, gender and fracture type. The Mantel-Haenszel OR will be reported. Stratum-specific ORs will be generated and tested for homogeneity across strata using the Breslow-Day test. ${ }^{42}$ If the result is significant, separate ORs for each stratum will be reported.

The ability to walk independently at each time point (60, 180 and 365 days) will be analysed using multivariable logistic regression models that control for other covariates, including stratification factors (site, gender and fracture type), and baseline variables that have potential association with the outcome, with particular attention to any such variable that appears imbalanced between treatment groups. Generalised linear mixed models will be used to perform a repeated measures analysis, looking at the ability to walk at 60, 180 and 365 days. We will use residual (or restricted) maximum likelihood methods for parameter estimates and significance testing. A function of time and the covariates aforementioned will be included as fixed effects in the regression.

The analytical approaches specified for the primary outcome will also be used for the binary secondary outcomes, including need for assistive devices for walking, postoperative delirium, mortality, return to the prior residence, occurrence of any major in-hospital complication. Continuous secondary outcomes including WHODAS V.2.0 score, pain scale values, cognitive function score and patient satisfaction scores will be compared between treatment groups using analysis of variance adjusting for the above stratification factors.

Missing data: We will evaluate the amount, reasons for and patterns of missing data, with a particular attention to the lost to follow-up data, in primary and secondary outcomes. Primary and secondary analyses will assume missing values are 'at random', relative to other data that we have collected; if the reasons for missing values suggest that the missingness is 'non-ignorable' (ie, not at random), we will develop models for missingness (eg, a selection model where the risk for drop-out depends on some clinical response) and use these models to help us 
assess the potential impact of missing data on our results. $^{43}$ We will also do a 'worst case scenario' sensitivity analysis, that is, all missing 60-day values in one treatment group will be replaced with the worst outcome and those in the other group with the best outcome.

Heterogeneity of treatment effects: Subgroup comparisons will be conducted if any treatment-covariate interactions are at least suggestive $(p<0.20)$ and sample sizes and numbers of events within these subgroups are sufficient for analysis. Secondary outcomes also will be assessed for heterogeneity of treatment effects. If there is a treatment difference together with evidence of heterogeneity, the relevant covariates and interaction terms will be added to the relevant regression models for formal significance testing. For the primary outcome, we plan for analyses of treatment effects within prespecified subgroups potentially defined by: (1) fracture type; (2) gender; (3) prefracture level of overall disability; (4) prefracture disability in locomotion; (5) age category; (6) baseline cognitive status; (7) surgical procedure; (8) baseline pulmonary disease; (9) baseline cardiac disease; (10) nursing home versus non-nursing home residence prior to fracture. These analyses will all be considered exploratory.

Data linkages: Necessary identifying data (ie, social security number, Medicare beneficiary number) will be obtained from consenting participants to facilitate data linkage to the NDI and to Medicare claims for planned analyses of survival data and healthcare usage data. Patients who do not provide these data will still be eligible to participate in this study with informed consent.

Interim analyses: Since both spinal and general anaesthesia are considered standard care for hip fracture surgery, we do not intend to consider early termination on the basis of efficacy data; however, interim efficacy data will be provided to the Data Safety Monitoring Board (DSMB) to permit benefit-to-risk assessments.

Data management: The Clinical Research Computing Unit of the University of Pennsylvania Center for Clinical Epidemiology and Biostatistics will serve as the REGAIN Data Coordinating Center and will provide a central location for data processing and management. All study data will be collected via an online data management system using the Oracle Remote Data Capture software, with encrypted transmission of remotely entered data. Separate data entry systems and study databases will be maintained for identifiable data required for patient follow-up, and de-identified clinical data; unique study identifiers will be assigned to each patient to allow for linkage across databases. Data will be stored on secure computing servers and will be restricted via password protections to only those individuals who are authorised to work on the trial. Specific privilege assignments within the database will also be employed to limit the types of data that authorised users may access to the minimum required by their role in the trial. Electronic audit trails will be used to capture and record changes to database contents automatically.

\section{Site training}

Training for REGAIN sites will be provided via: (1) in-person training meetings, including national kick-off events held in Philadelphia and Chicago in February and October 2016, for orientation to the study protocol and procedures; (2) online webinars for training and certification in the study data management system; and (3) self-learning activities for training and certification in study processes and selected study instruments. As necessary, site personnel may be required to undergo retraining, either through the online webinars or during site visits made by Coordinating Center staff.

Prior to initiation of data collection at a given site, all site personnel will be required to submit signed attestations of completion of required training tasks and to demonstrate proficiency in specific key competencies. Where relevant, site personnel will be required to demonstrate proficiency in data entry into the online data management system, with demonstration of competency of basic data entry and troubleshooting functions. For personnel completing 3D-CAM assessments, demonstration of proficiency will be required via satisfactory completion of assessments for three simulated patients using standardised web videos, with a correct overall diagnosis (delirium present/absent) and correct identification of all four features of CAM-defined delirium ${ }^{45}$ required for a passing score. For personnel completing data abstraction functions, certification demonstration of proficiency in abstraction of required data into study case report forms from two de-identified intraoperative anaesthesia records.

\section{Monitoring}

The REGAIN study monitoring plan incorporates remote and on-site monitoring appropriate for the risk level involved in the trial. ${ }^{46}$ Remote monitoring will take place via regular communication between Clinical Coordinating Center staff and recruiting site staff via email, conference call and web conference; communications will take place at regular intervals to review progress and identify issues, and as needed to address identified concerns. Sites will be provided with interval performance reports on recruitment progress, consent rates, data completeness and data timeliness.

Additional remote monitoring activities will include review and reabstraction of selected chart data from participating sites by trained staff within the Clinical Coordinating Center. Site personnel will de-identify portions of the medical record for the first three randomised patients and as needed thereafter and transmit them to the University of Pennsylvania for reabstraction. Identified discrepancies will be reported back to site staff for resolution and continuous quality improvement. Additional documents may be requested on an as-needed basis for monitoring purposes. Coordinating Center staff will also regularly review the completeness and timeliness of all data entries, and adherence to treatment in each study arm for each site. 
Non-adherence and data issues will be individually investigated and remediated as necessary.

The REGAIN study monitoring and quality assurance procedures will aim to ensure adherence to the assigned treatment for all patients and avoid cross-overs to comparator treatments. Remotely collected data will be reviewed on an ongoing basis to identify cross-over events; reasons for individual cross-overs are investigated, and sites will be required to file protocol deviation reports where appropriate. The importance of avoiding cross-overs will be stressed to site staff on a regular basis in monthly phone calls and all-investigator emails, and sites will be regularly counselled on the need for adherence to aspects of the protocol designed to limit crossovers. Prior to randomisation, site personnel will be required to verify that the treating anaesthesiologist believes the patient is suitable for randomisation and agrees deliver the assigned study treatment; further, site personnel will be encouraged to randomise the patient immediately prior to surgery in order to limit the possibility of cross-overs occurring due to changes in clinical status over time or related to changes in anaesthesia staffing.

Additional on-site or remote monitoring at participating sites will take place 1-2 times over the study period for review of the study regulatory binder for completeness and accuracy, review of consent documents, selected patient medical records for data completeness and accuracy, and on-site evaluation of adherence to study processes and procedures.

\section{Data and safety monitoring}

All serious adverse events, as well as all non-serious adverse events that are unexpected and judged to be related to the study treatment, will be recorded in the study database and reported as required to local IRBs and to the University of Pennsylvania IRB. Data and safety monitoring will be the responsibility of the study director/principal investigator (PI), the study biostatistician, site clinical directors and an independent DSMB selected by the study PI.

The DSMB roles, responsibilities and operating procedures are defined by the REGAIN DSMB charter. The DSMB will be composed of 5-7 independent, multidisciplinary experts who are not involved in the conduct of the study in any way; who do not have subordinate relationships with the PI or any member of the study team; and who are qualified through other experience or training to review the clinical and research data from the study. The DSMB will not be blinded to participant treatment assignment.

The DSMB met prior to the initiation of enrolment to review the protocol, the DSMB charter and reporting templates. Subsequent DSMB meetings will review the protocol, safety and adverse event data, available outcome data, and information on participant accrual and protocol compliance; these meetings will take place after randomisation of the first 100 patients and after randomisation of $1 / 4,1 / 2$ and $3 / 4$ of the total planned randomised sample. The DSMB will serve in an advisory capacity to the PI, and recommendations for protocol modifications or revisions to the informed consent document will be communicated directly to the study PI.

Study risks: The risks associated with this study are low. The risk of a breach of confidentiality is small and all possible efforts have been taken to ensure the security of study data and minimise the risks of accidental disclosure of identifiable data elements. The medical risks for participation in this study do not go beyond those risks typically associated with spinal or general anaesthesia as used in routine clinical care. Beyond the study consent, patients will also undergo standard procedural consent to discuss the risks and benefits of regional and general anaesthesia as per the standard of care at the local hospital.

\section{Ethics and dissemination}

Of currently approved US sites, seven have designated the University of Pennsylvania Perelman School of Medicine IRB as the IRB of record for this study. Recruitment began on 12 February 2016 and will continue through the end of 2019, with a target date for submission of the primary trial manuscript of 30 September 2020.

Protected health information will only be shared with research team members as required for completion of designated study tasks; patient contact information will be transmitted to the Clinical Coordinating Center for follow-up via secure network servers as described above. Electronic data and demographic information will be accessed only as necessary for completion of study follow-up tasks, and will not be printed or transferred from the study server to any secondary media. Lists will be maintained identifying all team members with access to identifiable study data, and dates and times of database access by team members will be logged.

\section{Engagement and dissemination}

Patient and stakeholder partners will be involved at all stages of the REGAIN trial. The lead patient partner for REGAIN is the Center for Advocacy for the Rights and Interests of the Elderly (Philadelphia, Pennsylvania, USA); in addition, the REGAIN trial leadership receives input from a patient partner panel which includes CARIE staff and seven lay members, including patients, caregivers and community members. Patient partners reviewed and provided input of the study protocol, and will meet at regular intervals over the course of the study to receive updates on study progress and provide ongoing input related to study conduct and interpretation and dissemination of results.

The lead stakeholder partner is the Gerontological Society of America (GSA; Washington DC, USA); in addition, the REGAIN trial leadership will receive input from a stakeholder partner panel which will be convened by GSA staff and will include representatives from 
relevant national stakeholder organisations. Stakeholder partners will help design and implement dissemination strategies for study findings to relevant lay and professional audiences.

Dissemination plans include presentations at local, national and international scientific conferences, and publications in scientific and lay journals. Study results will also be presented by study staff to affected populations within communities served by participating trial sites.

\section{CONCLUSIONS}

The REGAIN trial is a multicentre trial that will randomise 1600 older adults to receive either spinal anaesthesia or general anaesthesia for hip fracture surgery. Through an innovative pragmatic design and implementation across a broad range of geographic locations, hospital types and practice settings, REGAIN will yield important new information to directly impact the care and outcomes of the more than 1.6 million patients undergoing surgery for hip fracture each year worldwide.

\section{Author affiliations \\ ${ }^{1}$ Department of Anesthesiology and Critical Care, Perelman School of Medicine at the University of Pennsylvania, Philadelphia, Pennsylvania, USA \\ ${ }^{2}$ Leonard Davis Institute for Health Economics, The University of \\ Pennsylvania, Philadelphia, Pennsylvania, USA \\ ${ }^{3}$ Department of Internal Medicine, Division of Geriatric Medicine, Perelman School of Medicine at the University of Pennsylvania, Philadelphia, \\ Pennsylvania, USA \\ ${ }^{4}$ Center for Perioperative Outcomes Research and Transformation (CPORT), University of Pennsylvania, Philadelphia, Pennsylvania, USA \\ ${ }^{5}$ Department of Biostatistics and Epidemiology, Perelman School of Medicine at the University of Pennsylvania, Philadelphia, Pennsylvania, USA \\ ${ }^{6}$ Department of Anesthesiology, Johns Hopkins Bayview Medical Center, Baltimore, Maryland, USA \\ ${ }^{7}$ Department of Epidemiology and Public Health, University of Maryland School of Medicine, Baltimore, Maryland, USA \\ ${ }^{8}$ Department of Medicine, Division of General Internal Medicine, Rutgers Robert Wood Johnson Medical School, New Brunswick, New Jersey, USA}

\section{Twitter Follow Mark Neuman at @mdneuman}

Collaborators REGAIN Investigators: MDN, JLC, FES, JSM, SSE, RF, Lee A Fleisher, Denise Orwig, Samir Mehta, Edward Marcantonio, Nabil Elkassabany, J Sanford Schwartz, Steven L Kates, Lakisha Gaskins, Brittany Montgomery, Elaine Spangler, Denise Cifelli, Christopher Helker, Ro-Pauline Doe, Nyra Williams, Jim Datillo, Ann Tierney, Kamen Vlassakov, Lauren Gavin, Daniel Sessler, Wael Ali Sakr Esr, Sabry Ayad, Manal Hassan, Mark Cipolle, Aryeh Shander, Gregg Lobel, Thomas Looke, Courtland Lewis, Richard Sheppard, Mohamed Rida Alsaden, Jean-Pierre Ouanes, Robert Hymes, David Sanders, Alexander Hannenberg, Antoun Nader, Tiffany Tedore, Mitchell Marshall, Steven Papp, Derek Donegan, Sanjib Adhikary, Karen Troxell, Geza Kiss, Barry Perlman, Stephen Choi, Syed Azim, Meera Gonzalez, Christopher Haydel, Ki Jinn Chin, Joshua Sappenfield, Matthew Warrick, Mark Powell, Derek Dillane, Jonathan Waters, Timothy Dominick, A Jared Dabiri, Wade Weigel, J Douglas Jaffe, Shamsuddin Akhatar, Greg O'Neill, Patricia D'Antonio, Diane Menio, Jennifer Hruslinski, Sandy Diamond, Catherine Ferrick, James Hunt, Christine Langlois, Donna Nurick, Bernice Soffer, Linda Stojak, Eleanor Sokoloff.

Contributors MDN, SSE, FES, RF, JSM and JLC each made substantial contributions to the conception or design of the study protocol. MDN conceived the overall study and wrote the first draft of the protocol and this manuscript. FES, JSM, SSE and JLC provided critical input regarding the design of the study intervention, study outcomes and study procedures; MDN, SSE and RF designed the data analysis and management plan. MDN, SSE,
FES, RF, JSM and JLC revised the protocol critically for important intellectual content and approved the final version to be published. MDN, SSE, FES, RF, JSM and JLC agree to be accountable for all aspects of the work in ensuring that questions related to the accuracy or integrity of any part of the work are appropriately investigated and resolved.

Funding This work was funded through a Patient-Centered Outcomes Research Institute (PCORI) Award (PCS 1406-18876).

Disclaimer The views, statements and opinions presented in this work are solely the responsibility of the author(s) and do not necessarily represent the views of the PCORI, its Board of Governors or Methodology Committee.

Competing interests MDN, SSE and RF report grants from PCORI, during the conduct of the study. MDN reports grants from National Institutes of Health, outside the submitted work. JSM, JLC and FES report grants from University of Pennsylvania subcontract as study investigator, during the conduct of the study. JSM reports personal fees from Novartis, personal fees from Scholar Rock, personal fees from Ammonett LLC, personal fees from Viking, personal fees from Sanofi, outside the submitted work.

Ethics approval University of Pennsylvania Perelman School of Medicine IRB and the IRBs or Research Ethics Boards (REBs) of 26 participating US institutions and 2 Canadian institutions.

Provenance and peer review Not commissioned; externally peer reviewed.

Open Access This is an Open Access article distributed in accordance with the Creative Commons Attribution Non Commercial (CC BY-NC 4.0) license, which permits others to distribute, remix, adapt, build upon this work noncommercially, and license their derivative works on different terms, provided the original work is properly cited and the use is non-commercial. See: http:// creativecommons.org/licenses/by-nc/4.0/

\section{REFERENCES}

1. Brauer CA, Coca-Perraillon M, Cutler DM, et al. Incidence and mortality of hip fractures in the United States. JAMA 2009;302:1573-9.

2. Blackman DK, Kamimoto LA, Smith SM. Overview: surveillance for selected public health indicators affecting older adults-United States. MMWR CDC Surveill Summ 1999;48:1-6.

3. Haentjens $P$, Autier $P$, Barette $M$, et al. Survival and functional outcome according to hip fracture type: a one-year prospective cohort study in elderly women with an intertrochanteric or femora neck fracture. Bone 2007;41:958-64.

4. Leslie WD, O'Donnell S, Jean S, et al. Trends in hip fracture rates in Canada. JAMA 2009;302:883-9.

5. Tajeu GS, Delzell E, Smith W, et al. Death, debility, and destitution following hip fracture. J Gerontol A Biol Sci Med Sci 2014;69:346-53.

6. Nahm ES, Resnick B, Orwig D, et al. Exploration of informal caregiving following hip fracture. Geriatr Nurs 2010;31:254-62.

7. Saltz C, Zimmerman S, Tompkins C, et al. Stress among caregivers of hip fracture patients. J Gerontol Soc Work 1999;30:167-81.

8. Braithwaite RS, Col NF, Wong JB. Estimating hip fracture morbidity, mortality and costs. J Am Geriatr Soc 2003;51:364-70.

9. Burge R, Dawson-Hughes $\mathrm{B}$, Solomon $\mathrm{DH}$, et al. Incidence and economic burden of osteoporosis-related fractures in the United States, 2005-2025. J Bone Miner Res 2007;22:465-75.

10. Boulton C, Currie C, Griffiths R, et al. National hip fracture database Anaesthesia sprint audit of practice. London: Royal College of Physicians, 2014.

11. Neuman MD, Rosenbaum PR, Ludwig JM, et al. Anesthesia technique, mortality, and length of stay after hip fracture surgery. JAMA 2014;311:2508-17.

12. White SM, Griffiths R, Holloway J, et al. Anaesthesia for proximal femoral fracture in the UK: first report from the NHS Hip Fracture Anaesthesia Network. Anaesthesia 2010;65:243-8.

13. Guay J, Parker MJ, Gajendragadkar PR, et al. Anaesthesia for hip fracture surgery in adults. Cochrane Database Syst Rev 2016;2: CD000521.

14. Parker MJ, Handoll HH, Griffiths R. Anaesthesia for hip fracture surgery in adults. Cochrane Database Syst Rev 2004(4):CD000521.

15. Murthy S, Hepner DL, Cooper Z, et al. Controversies in anaesthesia for noncardiac surgery in older adults. Br J Anaesth 2015;115(Suppl 2):ii15-25.

16. Roberts KC, Brox WT. AAOS clinical practice guideline: management of hip fractures in the elderly. J Am Acad Orthop Surg 2015;23:138-40. 
17. Luger TJ, Kammerlander C, Gosch M, et al. Neuroaxial versus general anaesthesia in geriatric patients for hip fracture surgery: does it matter? Osteoporos Int 2010;21(Suppl 4):S555-72.

18. U.K. National Clinical Guideline Centre. The management of hip fracture in adults. London, UK: National Clinical Guideline Centre, 2011. http://www.ncgc.ac.uk

19. Thorpe KE, Zwarenstein M, Oxman AD, et al. A pragmaticexplanatory continuum indicator summary (PRECIS): a tool to help trial designers. J Clin Epidemiol 2009;62:464-75.

20. Ustun T, Kostanjsek N, Chatterji S. Measuring health and disability: manual for WHO disability assessment schedule (WHODAS 2.0). Geneva: World Health Organization, 2010.

21. Shulman MA, Myles PS, Chan MT, et al. Measurement of disabilityfree survival after surgery. Anesthesiology 2015;122:524-36.

22. Katzman R, Brown T, Fuld $\mathrm{P}$, et al. Validation of a short orientation-memory-concentration test of cognitive impairment. Am J Psychiatry 1983;140:734-9.

23. Kawas C, Karagiozis H, Resau L, et al. Reliability of the Blessed Telephone Information-Memory-Concentration Test. J Geriatr Psychiatry Neurol 1995;8:238-42.

24. Palihnich K, Inouye SK, Marcantonio ER. The 3D CAM training manual for research. 2nd edn. Boston: Hospital Elder Life Program, 2014.

25. Marcantonio ER, Ngo LH, O'Connor M, et al. 3D-CAM: derivation and validation of a 3-minute diagnostic interview for CAM-defined delirium: a cross-sectional diagnostic test study. Ann Intern Med 2014;161:554-61.

26. Cleeland CS, Ryan KM. Pain assessment: global use of the Brief Pain Inventory. Ann Acad Med Singapore 1994;23:129-38.

27. Keller S, Bann CM, Dodd SL, et al. Validity of the brief pain inventory for use in documenting the outcomes of patients with noncancer pain. Clin J Pain 2004;20:309-18.

28. Smith BW, Dalen J, Wiggins K, et al. The brief resilience scale: assessing the ability to bounce back. Int J Behav Med 2008;15:194-200.

29. Chernik DA, Gillings D, Laine $\mathrm{H}$, et al. Validity and reliability of the Observer's Assessment of Alertness/Sedation Scale: study with intravenous midazolam. J Clin Psychopharmacol 1990;10:244-51.

30. Carson JL, Terrin ML, Magaziner J, et al. Transfusion trigger trial for functional outcomes in cardiovascular patients undergoing surgica hip fracture repair (FOCUS). Transfusion 2006;46:2192-206.

31. Gill TM. Disentangling the disabling process: insights from the precipitating events project. Gerontologist 2014;54:533-49.
32. Juster FT, Suzman R. An overview of the health and retirement study. J Hum Res 1995;30:S7-S56.

33. Carson JL, Terrin ML, Noveck H, et al. Liberal or restrictive transfusion in high-risk patients after hip surgery. $N$ Engl J Med 2011;365:2453-62.

34. Bauer M, Böhrer $\mathrm{H}$, Aichele $\mathrm{G}$, et al. Measuring patient satisfaction with anaesthesia: perioperative questionnaire versus standardised face-to-face interview. Acta Anaesthesiol Scand 2001;45:65-72.

35. Fuzier R, Bataille B, Fuzier V, et al. Spinal anesthesia failure after local anesthetic injection into cerebrospinal fluid: a multicenter prospective analysis of its incidence and related risk factors in 1214 patients. Reg Anesth Pain Med 2011;36:322-6.

36. Munhall RJ, Sukhani R, Winnie AP. Incidence and etiology of failed spinal anesthetics in a university hospital: a prospective study. Anesth Analg 1988;67:843-8.

37. Fettes PD, Jansson JR, Wildsmith JA. Failed spinal anaesthesia: mechanisms, management, and prevention. $\mathrm{Br} J$ Anaesth 2009;102:739-48.

38. Andrews G, Kemp A, Sunderland M, et al. Normative data for the 12 item WHO Disability Assessment Schedule 2.0. PLOS ONE 2009;4: e8343.

39. Saks EB, Dunn LB, Wimer J, et al. Proxy consent to research: the legal landscape. Yale J Health Policy Law Ethics 2008;8:37-92.

40. Matts JP, Lachin JM. Properties of permuted-block randomization in clinical-trials. Control Clin Trials 1988;9:327-44.

41. Lachin JM, Matts JP, Wei LJ. Randomization in clinical trials: conclusions and recommendations. Control Clin Trials 1988;9:365-74.

42. Breslow N, Day N. Statistical methods in medical research, volume 1: analysis of case-control studies. IARC Scientific Publications. Lyon, France: International Agency for Research on Cancer, 1980.

43. Verbeke $\mathrm{G}$, Molenberghs $\mathrm{G}$, Thijs $\mathrm{H}$, et al. Sensitivity analysis for nonrandom dropout: a local influence approach. Biometrics 2001;57:7-14

44. Molenberghs $\mathrm{G}$, Thijs $\mathrm{H}$, Jansen I, et al. Analyzing incomplete longitudinal clinical trial data. Biostatistics 2004;5:445-64.

45. Inouye SK, van Dyck $\mathrm{CH}$, Alessi CA, et al. Clarifying confusion: the confusion assessment method. A new method for detection of delirium. Ann Intern Med 1990;113:941-8.

46. U.S. Food and Drug Administration. Guidance for industry: oversight of clinical investigations-a risk-based approach to monitoring. Washington DC: US Department of Health and Human Services, 2013. 